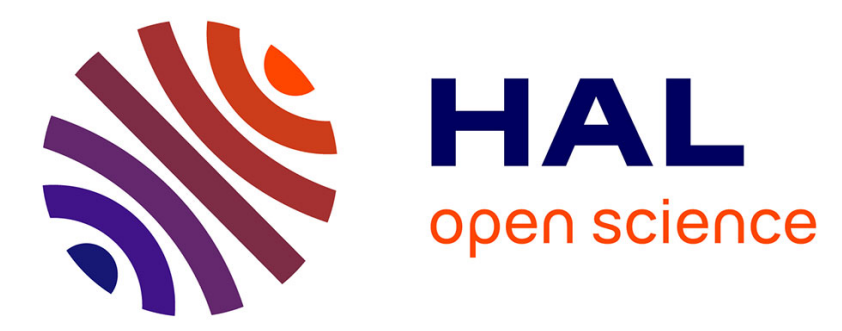

\title{
Vaccination par le BCG : enquête auprès d'enfants de moins de 5 ans consultant dans un service d'urgences hospitalières
}

A Vernaz, Jean Gaudart, K Sallah, L Casanova, A Debroise, R Laporte, P Minodier

\section{To cite this version:}

A Vernaz, Jean Gaudart, K Sallah, L Casanova, A Debroise, et al.. Vaccination par le BCG: enquête auprès d'enfants de moins de 5 ans consultant dans un service d'urgences hospitalières. Archives de Pédiatrie, 2014, 10.1016/j.arcped.2014.02.030 . hal-01307530

\section{HAL Id: hal-01307530 \\ https://hal-amu.archives-ouvertes.fr/hal-01307530}

Submitted on 26 Apr 2016

HAL is a multi-disciplinary open access archive for the deposit and dissemination of scientific research documents, whether they are published or not. The documents may come from teaching and research institutions in France or abroad, or from public or private research centers.
L'archive ouverte pluridisciplinaire HAL, est destinée au dépôt et à la diffusion de documents scientifiques de niveau recherche, publiés ou non, émanant des établissements d'enseignement et de recherche français ou étrangers, des laboratoires publics ou privés.

\section{(ㅇ)(1) $\$$}

Distributed under a Creative Commons Attribution - NonCommercial - NoDerivatives 44.0 
Reçu le :

3 avril 2013

Accepté le :

12 février 2014

Disponible en ligne

8 avril 2014
CrossMark

\section{Disponible en ligne sur}

\section{ScienceDirect}

www.sciencedirect.com

\section{Vaccination par le BCG : enquête auprès d'enfants de moins de 5 ans consultant dans un service d'urgences hospitalières}

\author{
BCG vaccination: Survey among children less than 5 years of \\ age in an emergency department
}

\author{
A. Vernaz ${ }^{a}$, J. Gaudart ${ }^{b}$, K. Sallah ${ }^{b}$, L. Casanova ${ }^{c, d}$, A. Debroise ${ }^{a}$, \\ R. Laporte ${ }^{a}$, P. Minodier ${ }^{a, *}$ \\ ${ }^{a}$ Service d'urgences pédiatriques, CHU Nord, chemin des Bourrelly, 13920 Marseille cedex 15, \\ France \\ ${ }^{\mathrm{b}}$ Aix-Marseille université, UMR912 (Inserm/IRD/AMU), 13005 Marseille, France \\ ' Département de médecine générale, faculté de médecine, université Aix-Marseille, 27, \\ boulevard Jean-Moulin, 13005 Marseille, France \\ d ORS PACA, observatoire régional de santé, 13009 Marseille, France
}

\section{Summary}

France is a country with a low incidence of tuberculosis. However, there are important local variations: some parts of the city of Marseilles (southern France) presented a yearly incidence greater than $30 / 100,000$ in 2010 . The main goal of this study was to evaluate the BCG vaccine coverage among at-risk children younger than 5 years consulting in one of the city's pediatric emergency departments.

Material and methods. This descriptive study took place in February 2013 and used a semi-directive questionnaire distributed to parents.

Results. One hundred and thirty-five children were included, $98(72.6 \%)$ were considered as being at risk of tuberculosis and among them 75 (76.5\%, $95 \%$ CI [68.0-85.1]) were vaccinated with BCG. Parents' knowledge of tuberculosis was relevant in $48 \%$ of the respondents. Only $19 \%$ of the parents reported that BCG protects against tuberculosis, but $73 \%$ were in favor of this vaccination. Two criteria significantly increased vaccine coverage among at-risk children: birth in Marseilles and age more than 6 months on the consultation day. The child living in a poor family and type of medical followup did not significantly influence BCG vaccine coverage.

Conclusion. BCG coverage was high in at-risk children younger than 5 years born in Marseilles. Cooperation between private physicians, maternity hospitals and mothers, and children's public health services probably facilitates this high level of protection. (c) 2014 Elsevier Masson SAS. All rights reserved.

\section{Résumé}

Malgré une faible incidence de la tuberculose en France, il existe de grandes variations à l'échelle communale : certains quartiers de Marseille présentent ainsi une incidence annuelle supérieure à 30/ 100 000. Le but de notre étude était d'évaluer la couverture vaccinale par le vaccin de Calmette et Guérin (BCG) des enfants à risque âgés de moins de 5 ans consultant dans un service d'urgences pédiatriques. Matériel et méthodes. Cette étude descriptive a été réalisée en février 2013 à l'aide d'un questionnaire semi-directif proposé aux parents.

Résultats. Cent-trente-cinq enfants ont été inclus, 98 (72,6\%) étaient considérés à risque de tuberculose et parmi ces derniers 75 (76,5\%, IC $95 \%$ [68,0-85,1]) étaient vaccinés par le BCG. Seuls $48 \%$ des parents connaissaient la tuberculose et $19 \%$ savaient que le BCG protégeait contre cette maladie, mais $73 \%$ étaient favorables à ce vaccin. Deux paramètres étaient liés à la vaccination des enfants à risque : une naissance à Marseille et un âge supérieur à 6 mois le jour de la consultation. La précarité des conditions de vie et le type de suivi médical n'influençaient pas significativement la couverture vaccinale des enfants à risque.

Conclusion. En 2013, la couverture vaccinale par le BCG des enfants de moins de 5 ans nés à Marseille et à risque de tuberculose était correcte mais perfectible. Une coopération entre médecins de ville, maternités et services de protection maternelle et infantile (PMI) permet probablement d'obtenir cette bonne couverture vaccinale. (C) 2014 Elsevier Masson SAS. Tous droits réservés.

\footnotetext{
* Auteur correspondant.

e-mail : philippe.minodier@ap-hm.fr (P. Minodier). 


\section{Introduction}

Malgré une nette diminution de son incidence depuis plusieurs années, la tuberculose reste un problème majeur de santé publique dans le monde. En 2011, on estimait à 8,7 millions le nombre de nouveaux cas et à 1,4 millions le nombre de décès liés à cette maladie ; 500 000 nouveaux cas et 64000 décès concernaient des enfants de moins de 15 ans [1]. La France fait partie des pays à faible incidence de tuberculose (8,1/100 ooo en 2010) [2]. Il existe cependant de fortes disparités selon les populations (incidences chez les sans domicile fixe et chez les personnes nées à l'étranger respectivement de 155 et 36 cas/100 000/an) et selon les régions (I'île-de-France, la Guyane et Mayotte ayant les plus fortes incidences). La région Provence-Alpes-Côte d'Azur est la $5^{\mathrm{e}}$ région la plus touchée de France, avec 8 nouveaux cas/ 100 ooo habitants/an en 2010 [2]. La majeure partie des cas se concentre dans les Bouches du Rhône (incidence de 9,5/ 100 000/an en 2010) et plus particulièrement dans certains arrondissements de la ville de Marseille où les incidences sont supérieures à 30 cas/100 000/an [3].

En France, depuis juillet 2007, la vaccination par le vaccin de Calmette et Guérin (BCG) n'est plus obligatoire pour l'entrée en collectivité des enfants [4], mais elle est fortement recommandée pour les enfants à risque élevé de tuberculose [5]. La couverture vaccinale (CV) de ces enfants à risque nés après la suspension de l'obligation vaccinale a été évaluée par plusieurs études nationales [6-8], et variait entre $44 \%$ (médecins généralistes du réseau Sentinelle) et $90 \%$ (médecins de protection maternelle et infantile [PMI] d'île-de-France). Elle était généralement plus élevée en île-de-France qu'en province [9]. À Marseille, une étude menée en 2009 dans le service hospitalier d'accueil des urgences pédiatriques (SAUP) du centre-ville avait trouvé une CV de $74 \%$ chez les nourrissons à risque [10]. L'hôpital Nord, situé dans des quartiers défavorisés à forte population d'origine étrangère, a mis en place en 2007 un programme de vaccination des nouveau-nés à risque dès la maternité. Cette initiative a permis de maintenir une bonne CV des nourrissons à risque nés à la maternité a près le changement de politique vaccinale ( $C V$ de $88 \%$ à l'âge de 3 mois), en compensant la baisse de la vaccination en médecine de ville [11]. L'objectif principal de notre étude était d'évaluer la CV par le BCG chez les enfants à risque de moins de 5 ans consultant aux urgences pédiatriques de l'hôpital Nord. Elle visait secondairement à analyser la connaissance et l'opinion des parents à propos du BCG, la tolérance de ce vaccin et les facteurs pouvant influencer la CV.

\section{Matériel et méthodes}

\subsection{Cadre de l'étude}

L'hôpital Nord est l'un des 2 établissements de l'assistance publique des hôpitaux de Marseille recevant des enfants. Le
SAUP du site a réalisé près de 30000 consultations médicochirurgicales d'enfants de moins de 15 ans en 2011. Il est situé dans les quartiers nord de la ville, arrondissements à faible niveau socio-économique [12] qui présentent une natalité élevée et une faible installation médicale en ville. Son bassin de recrutement concerne les arrondissements du nord de Marseille et les communes limitrophes.

II s'est agi d'une étude descriptive non interventionnelle. Le recueil de données s'est déroulé entre le 28 janvier et le 8 février 2013, pendant 9 jours, de $9 \mathrm{~h}$ à $18 \mathrm{~h}$, au sein de ce service. L'étude a fait l'objet d'une autorisation de la Commission nationale de l'informatique et des libertés (numéro de déclaration $1636568 \mathrm{vo})$.

\subsection{Population étudiée}

Un questionnaire semi-directif sur le BCG a été proposé aux parents des enfants nés après le $1^{\text {er }}$ août 2007 consultant au SAUP durant la période de l'étude. Leur consentement écrit était nécessaire à l'inclusion. N'ont pas été inclus : les enfants non accompagnés d'un de leur parent ou tuteur légal, ceux nécessitant une prise en charge immédiate, ceux dont les parents ont refusé de répondre au questionnaire et ceux dont les parents ne pouvaient pas répondre au questionnaire à cause de la barrière de la langue.

La taille de l'échantillon (134 enfants) avait été préalablement calculée en fonction de l'estimation de la proportion d'enfants à risque parmi les enfants venant consulter au SAUP (60\%) $[10,11]$, de celle de la CV des enfants à risque nés après juillet 2007 (70 \%) [10,11] et de la précision du chiffre de CV désirée (10\%). Les questions posées aux parents visaient :

- à évaluer leurs connaissances sur le BCG et la tuberculose ; - à identifier les facteurs de risque de tuberculose de leur enfant ;

- à déterminer le statut vaccinal de celui-ci et les raisons en cas de défaut de vaccination et ;

- à évaluer les conditions de réalisation et la tolérance du vaccin.

En fin d'entretien, un document d'information sur le BCG ainsi que le calendrier vaccinal 2012 simplifié de l'Institut national de prévention et d'éducation pour la santé [13] étaient distribués aux parents et explicités par l'investigateur.

\subsection{Définition des variables}

Pour définir les enfants à risque les critères du Haut conseil de la santé publique (HCSP), reproduits dans l'avis 2012 sur les recommandations vaccinales [5], ont été utilisés. Ont été considérés comme présentant des facteurs objectifs de risque de tuberculose les enfants :

- nés dans un pays de forte endémie tuberculeuse [5] ;

- dont au moins un des parents était originaire de l'un de ces pays ;

- ayant séjourné ou devant séjourner au moins un mois d'affilée dans l'un de ces pays ; 
- ayant des antécédents familiaux de tuberculose (collatéraux ou ascendants directs) ;

- en contact fréquent ou occasionnel avec une personne ayant eu la tuberculose après la naissance de l'enfant;

- résidant habituellement en Île-de-France, en Guyane ou à Mayotte.

La définition du HCSP prend également en compte un risque subjectif déterminé par le médecin sur des critères personnels. À la lumière d'un travail précédent sur la CV chez les

\begin{tabular}{|c|c|c|}
\hline \multirow[t]{2}{*}{$\begin{array}{l}\text { Caractéristiques des enfants } \\
\text { et de leurs parents }\end{array}$} & \multicolumn{2}{|l|}{$\begin{array}{l}\text { Échantillon } \\
\text { total, } n=135\end{array}$} \\
\hline & $n$ & $\%$ \\
\hline \multicolumn{3}{|l|}{ Âge des enfants } \\
\hline$<1$ ans & 47 & 35 \\
\hline 1 à 2 ans & 24 & 18 \\
\hline 2 à 3 ans & 24 & 18 \\
\hline 3 à 4 ans & 18 & 13 \\
\hline$>4$ ans & 22 & 16 \\
\hline \multicolumn{3}{|l|}{ Naissance et résidence des enfants } \\
\hline Né à Marseille & 97 & 72 \\
\hline Né à l'Hôpital Nord & 55 & 41 \\
\hline Vit dans les Bouches du Rhône & 133 & 99 \\
\hline Vit à Marseille & 88 & 65 \\
\hline $\begin{array}{l}\text { Vit dans les arrondissements } \\
\text { limitrophes de I'hôpital }\end{array}$ & 85 & 63 \\
\hline \multicolumn{3}{|l|}{ Parents } \\
\hline Âge des mères & $30(S D=0,44)$ & \\
\hline Âge des pères & $34(S D=0,63)$ & \\
\hline Mère sans emploi & 96 & 71 \\
\hline Père sans emploi & 33 & 24 \\
\hline Mère isolée & 26 & 19 \\
\hline $\begin{array}{l}\text { Au moins un parent né dans } \\
\text { un pays à risque }\end{array}$ & 59 & 44 \\
\hline $\begin{array}{l}\text { Enfant ayant déjà voyagé dans } \\
\text { un pays à risque }\end{array}$ & 75 & 56 \\
\hline $\begin{array}{l}\text { Un parent au moins sans } \\
\text { étude secondaire }\end{array}$ & 16 & 12 \\
\hline \multicolumn{3}{|l|}{ Logement de la famille } \\
\hline Nombre de personnes par pièce & $4,1(S D=1,3)$ & \\
\hline $\begin{array}{l}\text { Surface moyenne par membre } \\
\text { du foyer }\left(\mathrm{m}^{2} / \text { personne }\right)\end{array}$ & $19,4(S D=5,6)$ & \\
\hline Propriétaires & 29 & 22 \\
\hline Locataires & 96 & 71 \\
\hline Hébergés & 8 & 6 \\
\hline Vivant en squat & 1 & 1 \\
\hline \multicolumn{3}{|l|}{ Suivi médical des enfants } \\
\hline Médecin généraliste seul & 48 & 36 \\
\hline Pédiatre seul & 39 & 29 \\
\hline PMI seule & 13 & 10 \\
\hline Hôpital seul & 1 & 1 \\
\hline Plusieurs professionnels & 33 & 24 \\
\hline Aucun suivi & 1 & 1 \\
\hline
\end{tabular}

$S D$ : déviation standard; $P M I$ : protection maternelle infantile enfants en situation de précarité [14], nous avons défini ce risque en considérant :

- des facteurs de risque " subjectifs majeurs " :

habitat précaire : caravane, mobile home, squat,

aide médicale d'état ou absence de couverture sociale, absence d'emploi chez les deux parents ;

- des facteurs de risque "subjectifs mineurs":

- parents bénéficiaires de la couverture maladie universelle (CMU),

mère seule et sans emploi,

enfant ayant voyagé ou devant voyager dans un pays à risque moins d'un mois d'affilée avant l'âge de 5 ans, enfant ayant voyagé ou devant voyager dans un pays à risque moins d'un mois d'affilée pour aller voir de la famille ;

- des facteurs de risque "subjectifs accessoires ":

- enfant vivant dans un arrondissement de Marseille à risque $: 1^{\mathrm{er}}, 2^{\mathrm{e}}, 3^{\mathrm{e}}, 6^{\mathrm{e}}, 13^{\mathrm{e}}, 14^{\mathrm{e}}, 15^{\mathrm{e}}$,

enfant dont la famille était hébergée,

enfant vivant dans un habitat densément peuplé ( $\leq 15 \mathrm{~m}^{2}$ /personne).

Dans notre étude, ont été considérés à risque les enfants présentant au moins 1 facteur de risque "objectif ", ou au moins 1 facteur de risque " subjectif majeur ", ou au moins 2 facteurs de risque " subjectifs mineurs ", ou un facteur de risque "subjectif mineur " et au moins 1 facteur de risque " subjectif accessoire". Ont été considérés comme vaccinés les enfants qui disposaient d'une trace écrite du BCG sur le carnet de santé ou qui présentaient une cicatrice caractéristique du vaccin au niveau d'un deltoïde ou dont les parents se souvenaient que le vaccin avait été réalisé.

\subsection{Analyse des données}

Les données ont été colligées anonymement sur le logiciel Stata 11 pour Windows. L'analyse des résultats a été faite à l'aide du même logiciel, selon les modes de report appropriés (effectifs, extrêmes, moyennes, écart-types...). L'effet des variables catégorielles a été testé en utilisant le test du $\mathrm{Chi}^{2}$ ou le test exact de Fisher. L'analyse multi-variée a été réalisée à l'aide d'une régression logistique, permettant ainsi d'obtenir des Odds Ratios (OR) ajustés. Les tests et intervalles de confiance (IC) ont pris comme risque alpha $5 \%$.

\section{Résultats}

\subsection{Population étudiée}

Cent-trente-cinq enfants de moins de 65 mois ont été inclus sur les 145 (93\%) à qui le questionnaire avait été proposé. Aucun enfant n'a été exclu du fait de la barrière de langue, même ceux dont les parents ne parlaient pas français ayant pu être interrogés grâce à la traduction d'un accompagnant. L'âge médian des enfants inclus était de 21 mois 
Tableau II

Facteurs objectifs de risque de tuberculose.

Facteurs objectifs de risque

\begin{tabular}{rl}
$\boldsymbol{n}(\mathbf{1 3 5})$ & \% \\
\hline 1 & 0,7 \\
59 & 43,7 \\
41 & 30,4 \\
1 & 0,7 \\
3 & 2,2 \\
69 & 51,1
\end{tabular}

Enfant né dans un pays à risque

Un des parents né dans un pays à risque

Voyage de plus de 1 mois dans un pays à risque de tuberculose

Antécédents familiaux de tuberculose (collatéraux ou ascendants)

Cas de tuberculose dans l'entourage après la naissance de l'enfant avec contacts fréquents ou occasionnels

Au moins 1 facteur objectif de risque

Tableau III

Facteurs de risque subjectifs de tuberculose.

Facteurs subjectifs de risque

$n(/ 135)$

$\%$

Facteurs de risque subjectifs majeurs

Habitat précaire (caravane, mobile home, squat)

Aide médicale d'état ou aucune couverture sociale

Les 2 parents sans emploi

Au moins 1 facteur subjectif majeur de risque

Sans facteur objectif de risque

Facteurs de risque subjectifs mineurs

Couverture maladie universelle

Mère seule et sans emploi

Voyage dans un pays à risque $<1$ mois

Âge au premier voyage $\leq 5$ ans

Voyage pour visiter la famille

Au moins 2 facteurs subjectifs mineurs de risque

Sans facteur objectif ni subjectif majeur de risque

$\begin{array}{rr}5 & 3,7 \\ 3 & 2,2 \\ 28 & 20,7 \\ 31 & 23,0 \\ 13 & 9,6\end{array}$

Facteurs de risque subjectifs accessoires

Résidence dans un arrondissement à risque

Hébergés

Surface habitable $\leq 15 \mathrm{~m}^{2} /$ personne

1 seul facteur subjectif mineur de risque et au moins 1 facteur subjectif accessoire de risque

Sans facteur objectif ni subjectif majeur de risque

$\begin{array}{rr}62 & 45,9 \\ 21 & 15,6 \\ & \\ 27 & 20,0 \\ 29 & 21,5 \\ 43 & 31,9 \\ 14 & 10,4 \\ & \\ 85 & 63,0 \\ 8 & 5,9 \\ 24 & 22,4 \\ 29 & 21,5 \\ 2 & 1,5\end{array}$

$\left(25^{\mathrm{e}}\right.$ percentile $[\mathrm{p}]=7$ et $75^{\mathrm{e}} \mathrm{p}=40$ ) (tableau I). Cinquanteneuf enfants ( $44 \%$ ) avaient au moins un de leur parent né dans un pays à risque : Afrique du Nord (18\% des 270 parents, $n=48)$, archipel des Comores (9 $\%, n=25)$, Europe de l'est (3 $\%, n=9)$, Afrique sub-saharienne $(2 \%, n=6)$ ou MoyenOrient $(1 \%, n=2)$. Soixante-quinze enfants (56\%) devaient ou avaient déjà voyagé dans un pays à risque, principalement en Afrique du Nord (72 \%, $n=54)$ et dans l'archipel des Comores (19\%, $n=14)$. Au total, 98 enfants (73\%) ont été considérés à risque de tuberculose (tableaux II et III). Plus de la moitié des enfants ( $51 \%, n=69$ ) présentaient un facteur de risque objectif, soit $70 \%$ des enfants à risque.

\subsection{Couverture vaccinale par BCG}

Soixante-quinze des 98 enfants à risque avaient été vaccinés par le BCG, soit une CV des enfants à risque de 76,5\% (IC $95 \%$ $[68,0-85,1])$. Parmi les 89 enfants vaccinés 73 (82\%) l'étaient de façon certaine (BCG écrit sur le carnet de santé ou cicatrice vue) et 16 (18\%) l'étaient de façon probable (d'après le souvenir des parents). Si n'avaient été inclus que les enfants avec carnet de santé $(n=100)$, la CV des enfants à risque aurait été de 73,2 \% (52/71) (IC $95 \%$ [62,9-83,5]). D'autre part si parmi les 135 enfants nous avions considéré à risque uniquement les enfants présentant des facteurs objectifs ou subjectifs majeurs $(n=82)$ en gardant notre définition des enfants vaccinés (certains et probables), la CV aurait été de $78,1 \%(n=59)$ (IC $95 \%[69,1-87,1])$.

L'âge médian de vaccination pour l'ensemble des enfants (à risque ou non) pour lesquels nous disposions de cette donnée $(n=79)$, était de 1 mois $\left(25^{\mathrm{e}} \mathrm{p}=\right.$ o et $\left.75^{\mathrm{e}} \mathrm{p}=1\right)$. Chez les enfants à risque vaccinés dont on connaissait l'âge de vaccination $(n=66), 5 \%$ l'avaient été après 6 mois $(n=3)$. Les vaccinateurs étaient des pédiatres $(35 \%, n=31)$, des médecins de la PMI ( $27 \%, n=24)$, des maternités $(23 \%, n=20$ ) et des médecins généralistes ( $12 \%, n=11$ ). Parmi les enfants dont l'âge de vaccination était connu, 29 des 43 enfants nés à la maternité du centre hospitalier (67\%) avaient été vaccinés avant 1 mois contre $22 \%$ des enfants nés ailleurs $(8 / 36)$ ( $p<0,0001)$. Vingttrois enfants à risque n'étaient pas vaccinés : parmi eux, 10 avaient reçu des informations sur le BCG de la part d'un médecin. Le vaccin avait été proposé à 7 d'entre eux : la 


\begin{tabular}{|c|c|c|c|c|c|c|c|c|}
\hline \multicolumn{9}{|c|}{$\begin{array}{l}\text { Tableau IV } \\
\text { Critères influençant la couverture vaccinale par le BCG. }\end{array}$} \\
\hline \multirow[t]{2}{*}{ Critères } & \multirow{2}{*}{$\begin{array}{l}\text { Enfants à } \\
\text { risque } n=98 \\
n\end{array}$} & \multirow{2}{*}{$\begin{array}{l}\text { Enfants } \\
\text { vaccinés } n=75 \\
n(\%)\end{array}$} & \multicolumn{3}{|c|}{ Analyse uni-variée } & \multicolumn{3}{|c|}{ Analyse multi-variée } \\
\hline & & & OR brut & [IC $95 \%$ ] & $p$ & OR ajusté & [IC $95 \%$ ] & $p$ \\
\hline Naissance à Marseille & 79 & $67(84,8)$ & 7,68 & {$[2,56-23,03]$} & 0,001 & 7,89 & {$[2,25-27,69]$} & 0,001 \\
\hline Âge $>6$ mois & 73 & $60(82,2)$ & 3,08 & {$[1,13-8,36]$} & 0,008 & 5,04 & {$\left[1,45^{-17,56]}\right.$} & 0,011 \\
\hline Mère seule & 24 & $16(66,7)$ & 0,51 & {$[0,18-1,41]$} & 0,09 & 0,37 & {$[0,10-4,77]$} & 0,137 \\
\hline Connaissance du $\mathrm{BCG}^{\mathrm{a}}$ & 12 & $7(58,3)$ & 0,37 & {$[0,11-1,3]$} & 0,08 & 0,3 & {$[0,06-1,54]$} & 0,147 \\
\hline Habitat précaire ${ }^{b}$ & 5 & $2(40)$ & 0,18 & {$\left[0,03^{-1,17}\right]$} & 0,1 & 0,47 & {$[0,03-6,40]$} & 0,569 \\
\hline Voyage en pays à risque $^{c}$ & 71 & $58(81,7)$ & 2,62 & {$[0,98-7,03]$} & 0,053 & 1,31 & {$[0,34-4,99]$} & 0,691 \\
\hline Âge de la mère $<25$ ans & 18 & $11(61,1)$ & 0,39 & {$[0,13-1,17]$} & 0,902 & 1,1 & {$[0,25-4,77]$} & 0,902 \\
\hline
\end{tabular}

$B C G$ : vaccin de Calmette et Guérin ; OR : odd ratio ; IC : intervalle de confiance

${ }^{a}$ Le parent interrogé était capable de citer au moins une indication du BCG.

${ }^{b}$ Domicile en caravane, en mobile home ou en squat.

'Quelle que soit la durée du voyage.

vaccination avait été programmée 4 fois ; les parents avaient refusé le vaccin par peur des effets secondaires 3 fois.

\subsection{Connaissance et opinion des parents à propos du BCG}

Parmi les 135 familles interrogées, respectivement $44 \%$ $(n=60)$ et $53 \%(n=72)$ ont déclaré ne pas connaître la tuberculose ou la vaccination BCG. Soixante-cinq parents (48\%) avaient une notion de la maladie, 26 (19\%) savaient que le BCG protégeait de la tuberculose, et 23 (17\%) ont pu citer une indication du vaccin. En revanche, 95 parents (70\%) pensaient que le BCG devait être effectué à tous les enfants. Cinquante-deux parents (39\%) estimaient correctement le risque de tuberculose de leur enfant. Parmi les 93 parents qui connaissaient au moins une notion juste sur la tuberculose ou le BCG, 41 (44\%) estimaient correctement le risque de tuberculose encouru par leur enfant, contre seulement 11 (26\%) des 42 autres parents $(p=0,048)$. Soixante-sept parents (50\%) avaient une opinion favorable vis-à-vis des vaccins en général, 7 (5\%) avaient une opinion défavorable et 56 (42\%) avaient des réticences vis-à-vis de certains vaccins seulement. Le taux d'opinion favorable vis-à-vis du BCG était de $73 \%$ pour l'ensemble des parents $(n=93)$, et de $74 \%$ pour ceux des enfants à risque (72/98). Parmi les parents d'enfants vaccinés, $90 \%$ pensaient que le médecin avait eu raison d'effectuer le vaccin ( $n=80$ ) et $93 \%$ auraient accepté à nouveau la vaccination si elle était à refaire $(n=83)$.

\subsection{Tolérance du vaccin}

Vingt-trois enfants (26\%) avaient présenté des effets secondaires d'après les parents, dont 17 abcès locaux (74 \% des effets secondaires), 4 cicatrices chéloïdes, 3 adénites, 1 fièvre, 1 dissémination du BCG et 1 éruption supposée allergique. Cinquante-deux cicatrices (normales ou chéloïdes) ont été constatées parmi les 89 enfants vaccinés, soit une prévalence de $58 \%$.

\subsection{Facteurs influençant la couverture vaccinale}

Vingt-quatre paramètres pouvant influencer le statut vaccinal des enfants à risque ont été testés en analyse uni-variée. Parmi eux, trois avaient un impact significativement positif sur la CV des enfants à risque : une naissance ou une résidence à Marseille et un âge supérieur à 6 mois le jour de la consultation aux urgences. Une forte corrélation ayant été observé entre les 2 premiers critères $(p=0,58)$, nous avons choisi de ne nous intéresser qu'au lieu de naissance. Après vérification de l'absence de trop forte colinéarité, les 7 paramètres les plus significatifs $(p<0,1)$ ont été étudiés dans un modèle multi-varié : le lieu de naissance et l'âge de l'enfant influençaient toujours significativement la CV (tableau IV). Ainsi, la connaissance de la maladie ou du BCG par les parents ou leurs opinions vis-à-vis des vaccins, le type de suivi médical (PMI, médecin généraliste, pédiatre), les facteurs de risque objectifs de tuberculose (pays de naissance de l'enfant ou des parents, notion de voyage en pays d'endémie, contact de la famille avec un sujet tuberculeux) ou les facteurs de précarité des conditions de vie (couverture sociale, type d'habitat, famille monoparentale, maîtrise de la langue française par les parents, niveau d'études, taux d'emploi, âge maternel, difficultés financières pour l'achat des médicaments) ne modifiaient pas de façon significative la probabilité d'être vacciné.

\section{Discussion}

Cette étude, menée auprès d'enfants de moins de 5 ans consultant au SAUP d'un hôpital des quartiers défavorisés de Marseille confirme la CV des nourrissons à risque (73,9\%) observée dans un autre SAUP de la ville en 2009 [10]. De la même manière, la $\mathrm{CV}$ des enfants à risque nés à Marseille (85\%) se rapproche de celle observée dans la maternité de notre hôpital en 2007 (88 \%) [11]. Ces chiffres sont comparables ou sensiblement inférieurs à ceux constatés en île-deFrance (68-9o \%) $[6,7,9]$, et meilleurs que ceux rapportés pour l'ensemble des villes métropolitaines de province (44-62\%) 
[6-8]. On est frappé du taux particulièrement élevé (72,6 \%) d'enfants à risque de tuberculose dans l'étude, alors qu'ils représentent 18 à $31 \%$ des cas dans les séries concernant la France métropolitaine en dehors de l'île-de-France [6-8]. Le niveau économique de la ville peut-être une des explications. En 2001, la moitié des Marseillais déclarait un revenu inférieur à 12837 euros par unité de consommation, le revenu médian national étant de 14650 euros. Les arrondissements du nord de la ville, limitrophes de l'hôpital de notre étude $\left(14^{\mathrm{e}}\right.$ et $15^{\mathrm{e}}$ arrondissements), sont parmi les plus pauvres : plus de 1 personne sur 3 s'y déclarait chômeur en 1999 pour moins de 1 sur 4 dans toute la ville; plus de 1 famille sur 4 y est monoparentale pour 1 sur 5 en moyenne à Marseille; beaucoup de personnes peu ou pas diplômées, un pourcentage élevé d'employés et d'ouvriers ; un pourcentage de population étrangère de 2 à 3 fois plus élevé que la moyenne de la ville; $10 \%$ des ménages ne déclarant aucun revenu et disposant quasi exclusivement des prestations sociales comme source de revenu [12]. Un facteur important est également à mettre en lien avec le caractère subjectif du risque. Parmi les enfants à risque, $70 \%$ présentaient un facteur de risque objectif. Les autres enfants rentraient dans le cadre de ce que le HCSP définit comme une "situation jugée à risque par le médecin " [5]. Notre classification en risques subjectifs majeurs ou mineurs, certes critiquable, tentait de mieux rendre compte du risque réel, en associant les facteurs de risque entre eux, et en considérant par exemple qu'un enfant ayant pour seul critère de risque une $\mathrm{CMU}$ n'était pas en soi réellement exposé à la tuberculose. En ne prenant en compte que les indications objectives de vaccination et les facteurs de risque que nous avons définis comme "subjectifs majeurs", on aurait identifié 82 enfants à risque, contre 98 dans l'étude ( $84 \%)$. La CV chez ces enfants-là aurait été de $78 \%$.

La variable "âge supérieur à 6 mois lors de la consultation " avait été testée dans notre étude car elle avait été rapportée ailleurs comme positivement liée à la vaccination [8]. II est probable que le BCG soit différé chez certains enfants en raison de la peur des effets secondaires dans le plus jeune âge ou des difficultés de la technique vaccinale. En plus de faire courir un risque de contamination tuberculeuse à l'enfant, ce retard rend nécessaire l'intradermoréaction préalable à la vaccination. L'injection peut alors être délicate en raison de l'agitation du nourrisson, plus marquée après 6 mois. Cependant dans notre étude, seulement $5 \%$ des enfants à risque vaccinés l'avaient été après 6 mois. Dans la littérature, d'autres facteurs ont pu être associés à une plus grande probabilité d'être vaccinés [8] : des conditions de vie défavorables, un voyage prévu dans une région hautement endémique, la perception de la tuberculose par le médecin généraliste comme une maladie commune ou la bonne connaissance des recommandations vaccinales par les médecins. II est possible que ces 2 derniers facteurs concourent aussi à expliquer la bonne $\mathrm{CV}$ des enfants nés à Marseille, la tuberculose n'y étant pas rare.
Bien que $27 \%$ des parents eussent entendu parler d'une personne ayant eu la tuberculose dans leur entourage, très peu connaissaient vraiment cette maladie et le BCG, et encore moins les nouvelles indications. Une étude anglaise de 2007 avait évalué la connaissance des parents et des soignants sur le BCG [15]. Sur les 253 personnes interrogées (133 étaient des parents), $72 \%$ avaient entendu parler du BCG, mais seulement $6 \%$ des parents étaient au courant des nouvelles recommandations ciblées mises en place, et aucun n'avait pu donner toutes les indications vaccinales. Cependant notre étude n'a pas montré de lien entre la connaissance de la maladie par les parents et la CV. En effet, les parents avaient une bonne opinion du BCG (73\%) et seules 3 familles d'enfants à risque l'avaient refusé ( $4 \%$ ). Ainsi, bien que peu informés, les parents n'étaient globalement pas opposés au vaccin.

Une étude menée en 2009 indiquait comme principale raison d'absence de vaccin chez les enfants à risque l'incapacité du médecin traitant à identifier les enfants à risque (33\%) avant le refus des parents (11\%) [8]. Dans notre étude, le principal motif de non vaccination était l'absence d'information des parents, $57 \%$ des non vaccinés, taux comparable à celui (61\%) observé dans un autre hôpital marseillais [10]. L'enquête nationale menée en 2009 dans les PMI [7] a montré également que parmi les enfants à risque non vaccinés et antérieurement vus en consultation, le vaccin avait été proposé dans $86 \%$ des cas mais toujours différé. Dans cette étude, $96 \%$ des parents avaient accepté la vaccination. La réticence médicale plus que parentale était aussi mentionnée dans une autre série [6] : si $86 \%$ des médecins interrogés (généralistes et pédiatres) proposaient le BCG aux enfants à risque, seulement $55 \%$ des médecins ayant un exercice libéral exclusif faisaient de même, et l'acceptation parentale était toujours bonne (76\%). Dans notre étude, bien qu'il n'ait pas été mis en évidence de différence de $\mathrm{CV}$ selon le type de suivi médical, on peut noter que les médecins généralistes représentaient la classe de médecins la moins représentee parmi les vaccinateurs du BCG. Ainsi, améliorer la CV des enfants à risque implique de sensibiliser davantage les médecins libéraux, mais également de privilégier des actions coopératives entre la médecine de ville et les services de PMI ou les maternités [11,16].

La peur des effets secondaires est souvent avancée comme un facteur limitant la pratique de la vaccination. Observées chez $26 \%$ des enfants de notre série, les complications étaient le plus souvent locales, comme cela a été rapporté ailleurs $[17,18]$. En fait, le risque est souvent lié à un mésusage ou à une erreur technique, eux-mêmes favorisés par le manque de pratique $[18,19]$.

Un certain nombre de réserves peut être porté sur notre étude. Par exemple, en l'absence de carnet de santé ou de cicatrice visible, nous avions choisi de considérer les enfants comme vaccinés si les parents se souvenaient que leur enfant l'avait été. Une surestimation de la CV des enfants à risque est 
donc possible, les parents pouvant se tromper en confondant le BCG avec les autres vaccins courants. Cependant, en ne prenant en compte que les enfants avec BCG mentionné sur le carnet de santé, nous aurions obtenu une CV des enfants à risque similaire, de $73,2 \%$, et nous aurions exclu les enfants les plus précaires, qui consultent souvent sans carnet. D'autre part, la puissance de notre étude, conçue pour évaluer la CV, était probablement insuffisante pour définir clairement les facteurs en lien avec celle-ci. Enfin, notre évaluation de la tolérance du BCG était très subjective, se basant uniquement sur les dires des parents. En réalité cette partie de l'étude avait plus pour but de recueillir le ressenti des parents vis-à-vis du BCG que de réaliser une évaluation fiable du taux de prévalence des différents effets secondaires.

\section{Conclusion}

Les deux principaux enseignements de cette étude sur le BCG menée dans un service d'accueil des urgences pédiatriques sont :

- la CV importante des enfants à risque $(77 \%)$, proche de celle retrouvée en Île-de-France dans cette population et ;

- le nombre élevé d'enfants exposés au risque de tuberculose dans les quartiers défavorisés de Marseille (73\%).

Ce taux de protection reflète la mobilisation des pédiatres de la maternité, des médecins des services de PMI et des médecins traitants pour la vaccination et semble propre à la ville de Marseille, où la tuberculose est plus fréquente que dans le reste des Bouches du Rhône. Etre né à Marseille augmente en effet significativement la CV des enfants à risque. Une minorité d'enfants est cependant vaccinée après 6 mois leur faisant courir un risque d'être infecté par la tuberculose. Malgré des complications locales fréquentes (26\%) et une mauvaise connaissance de la maladie, les parents ne font pas obstacle à la vaccination par le BCG.

\section{Déclaration d'intérêts}

Les auteurs déclarent ne pas avoir de conflits d'intérêts en relation avec cet article.

\section{Références}

[1] OMS. Rapport 2012 sur la lutte contre la tuberculose dans le monde. Disponible sur internet : URL : http://www.who.int/ tb/publications/global_report/fr/index.html. [Consulté le 3 décembre 2012].

[2] Antoine $D$, Che $D$. Les cas de tuberculose déclarés en France en 2010. Bull Epidemiol Hebd 2012;24-5:285-7.

[3] Charlet F, Blanes de Canecaude J, Polignano S. La tuberculose déclarée dans les Bouches du Rhône. Année 2010. Principales données épidémiologiques. Agence régionale de santé Paca, 2011. Disponible sur internet : URL : http://www.ars.paca.sante. $\mathrm{fr} /$ fileadmin/PACA/Site Ars Paca/Nos missions/Etudes et publications/Tuberculose/Rapport la tuberculose declareedans_les_Bouches_du_Rhone_annee2010_arspaca_octobre 2011. pdf. [Consulté le 24 novembre 2012].

[4] Circulaire $\mathrm{N}^{\circ}$ DGS/RI1/2007/318 du 14 août 2007 relative à la suspension de l'obligation de vaccination par le BCG des enfants et adolescents. Disponible sur internet : URL : http://www.sante.gouv.fr/fichiers/bo/2007/07-09/a0090271. htm. [Consulté le 19 décembre 2012].

[5] InVS. Le Calendrier des vaccinations et les recommandations vaccinales 2012 selon l'avis du Haut Conseil de la santé publique. Bull Epidemiol Hebd 2012;14-15:161-88.

[6] Guthmann JP, De La Rocque F, Boucherat M, et al. Couverture vaccinale BCG en médecine libérale : premières données chez le nourrisson, sept mois après la levée de l'obligation vaccinale en France. Arch Pediatr 2009;16:489-95.

[7] Guthmann JP, Fonteneau L, Desplanques L, et al. Couverture vaccinale $B C G$ chez les enfants nés après la suspension de l'obligation vaccinale et suivis dans les PMI de France : enquête nationale 2009. Arch Pediatr 2010;17:1281-7.

[8] Rossignol L, Guthmann JP, Kernéis S, et al. Barriers to implementation of the new targeted BCG vaccination in France: a cross sectional study. Vaccine 2011;29:5232-7.

[9] Guthmann JP, Le Strat Y, Fonteneau L, et al. Déterminants socio-économiques des vaccinations BCG et pneumocoque chez les enfants de la région parisienne. Résultats de l'enquête VACSIRS, 2010. InVS 2013:44 pp. Disponible sur internet : URL : http://opac.invs.sante.fr/index.php? lvl=notice_display\&id= 11442. [Consulté le 11/11/2013].

[10] Danvin C, Bosdure E, Brémond V, et al. BCG et nourrissons à risque de tuberculose : étude de couverture vaccinale à Marseille après la levée d'obligation. Arch Pediatr 2010;17:1510-5.

[11] Parache C, Carcopino X, Gossot S, et al. Couverture vaccinale par le BCG ciblé en maternité avant et après la suspension de l'obligation vaccinale. Arch Pediatr 2010;17:359-65.

[12] Bras M. De forts contrastes de revenus entre les quartiers de Marseille. SUD INSEE l'essentiel 2004. Disponible sur internet: URL : http://www.insee.fr/fr/insee_regions/provence/themes/ sud_essentiel/sie76/sie76.pdf. [Consulté le 03.04.2013].

[13] Inpes. Calendrier vaccinal 2012 simplifié. Vaccination, où en êtes-vous ? Disponible sur internet : URL : http://www.inpes. sante.fr/CFESBases/catalogue/pdf/1099.pdf. [Consulté le 18/ 12/2012].

[14] Bouhamam N, Laporte R, Boutin A, et al. Précarité, couverture sociale et couverture vaccinale : enquête chez les enfants consultant aux urgences pédiatriques. Arch Pediatr 2012;19: 242-7.

[15] Gordon M, Roberts H, Odeka E. Knowledge and attitudes of parents and professionals to neonatal BCG vaccination in light of recent UK policy changes: a questionnaire study. BMC Infect Dis 2007;7:82.

[16] Dommergues MA, Robichon F, Soltane S, et al. Vaccination des nouveau-nés à risque élevé de tuberculose dans une maternité française. Arch Pediatr 2009;16:1270-5.

[17] Dommergues MA, De La Rocque F, Dufour V, et al. Enquête sur les effets secondaires locaux du vaccin intradermique BCG$\mathrm{SSI}{ }^{\circledR}$ chez l'enfant de moins de six ans en France. Arch Pediatr 2007;14:102-8.

[18] Chol C, Guy C, Jacquet A, et al. Complications of BCG vaccine $S \mathrm{SS}{ }^{\circledR}$ recent story and risk management plan: the French experience. Pharmacoepidemiol Drug Saf 2013;22:359-64.

[19] Benamar F, Loupi E. Mésusage et/ou surdosage de vaccin BCG : suivi et bilan de 4 ans de notification spontanée. Thérapie 2001;56:739-42. 